\title{
Pan-TRK Inhibitor ONO-7579
}

National Cancer Institute

\section{Source}

National Cancer Institute. Pan-TRK Inhibitor ONO-7579. NCI Thesaurus. Code C140379.

An orally bioavailable, selective pan-tropomyosin-related-kinase (tyrosine receptor kinase;

TRK) inhibitor, with potential antineoplastic activity. Upon oral administration, ON O-7579 specifically targets and binds to TRK and fusion proteins containing sequences from neurotrophic tyrosine receptor kinase (NTRK) types 1 (NTRK1; TrkA), 2 (NTRK2; TrkB), and 3 (NTRK3; TrkC). This inhibits neurotrophin-TRK interaction and TRK activation, thereby preventing the activation of downstream signaling pathways and resulting in both the induction of cellular apoptosis and the inhibition of cell growth in tumors that overexpress TRK and/or express NTRK fusion proteins. TRK, a family of receptor tyrosine kinases (RT Ks) activated by neurotrophins, is encoded by NTRK family genes.T he expression of either mutated forms of, or fusion proteins involving, NTRK family members results in uncontrolled TRK signaling, which plays an important role in tumor cell growth, survival, invasion and treatment resistance. 